\title{
Estimação de Clubes Municipais de Convergência no Brasil no Período de 2000 a $2010^{*}$
}

\section{Estimation of Municipal Convergence Clubs in Brazil from 2000 to 2010}

\author{
Sergiany da Silva Lima ${ }^{a}$ \\ Ana Urraca Ruizb
}

\begin{abstract}
Resumo: O objetivo desta pesquisa é estimar os clubes de convergência municipais do Brasil com base no modelo de crescimento neoclássico ampliado. A evidência empírica acerca da existência de equilíbrios múltiplos (clubes de convergência) sugere que há fortes diferenças de produtividade entre as funções de produção agregadas dos municípios. Para testar essa hipótese, são usados os métodos de misturas finitas (MMF) e equação threshold, dada a baixa consistência dos estimadores de convergência em um único equilíbrio (DURLAUF; JOHNSON, 1995). O MMF estima os clubes de convergência pela distribuição de probabilidade dos determinantes de crescimento econômico. O threshold identifica as diferenças de produtividade da função de produção agregada. Os resultados encontrados são consistentes com a teoria, pois constatam múltiplos equilíbrios em clubes de convergência municipais, assim como a persistência da desigualdade de renda no tempo. O MMF identifica os determinantes do crescimento econômico municipal por clube, explicitando as elasticidades da dotação de fatores e da difusão tecnológica por nível de desenvolvimento econômico. Os clubes threshold sugerem que o diferencial de produtividade se explica pelos diferenciais na eficiência do uso do capital e na absorção tecnológica. Os maiores efeitos e significâncias dos parâmetros estimados ocorrem nos clubes mais avançados.
\end{abstract}

Palavras-chave: Desigualdades persistentes. Múltiplos equilíbrios. Spillovers tecnológicos.

\begin{abstract}
This paper aims to estimate the municipal convergence clubs of Brazil. To do that, it uses a neoclassical growth model that includes absorption and technological diffusion. The empirical evidence on the very existence of multiple equilibria (convergence clubs) suggests that there are strong differences among the aggregate production functions of Brazilian municipalities. To test these hypotheses, we use a finite mixture methods (FMM) and threshold equation, under the assumption of low consistency of

\footnotetext{
* $\quad$ Nosso agradecimento especial à Fundação de Amparo à Ciência e Tecnologia de Pernambuco (Facepe) pelo apoio financeiro.

Universidade Federal Rural de Pernambuco, Unidade Acadêmica de Serra Talhada. Serra Talhada, Pernambuco, Brasil.

b Universidade Federal Fluminense, Programa de Pós-Graduação da Faculdade de Economia. Niterói, Rio de Janeiro, Brasil.
} 
the convergence estimators to a single equilibrium (DURLAUF; JOHNSON, 1995). The FMM estimates the convergence clubs by the probability distribution of the determinants of economic growth. The Threshold Method identifies the productivity differences of the aggregate production function. The results are consistent with the theory, since they show multiple balances in municipal convergence clubs and persistence of the inequalities of the income in time. The FMM identifies the economic growth determinants by club, explaining the elasticities of factor endowments and technological diffusion by level of economic development. The threshold clubs suggest that the productivity gaps are due to the differences of the efficiency of capital use and technological absorption. The greatest effects and significance of the estimated parameters occurred in the most advanced clubs.

Keywords: Persistent inequalities. Multiple equilibria. Technological spillovers.

JEL Classification: O47; O43; $\mathrm{O} 33$.

\section{1 lntrodução}

O crescimento econômico regional do Brasil é caracterizado por desigualdades persistentes com convergência de renda condicional ao equilíbrio individual das economias (AZZONI, 2001). Com isso, não há evidências de que a convergência condicional signifique uma redução das desigualdades regionais de renda no país. Uma forma de mostrar isso é mediante a teoria dos clubes de convergência de Quah (1996) e o conceito de polarização de Anderson, Linton e Leo (2012). Quando as economias são agrupadas em ricas e pobres, identifica-se um processo de divergência da renda agregada no Brasil, aumentando a desigualdade econômica entre economias avançadas e atrasadas (FIGUEIREDO; PORTO JÚNIOR, 2015).

A renda de um município mede o valor da produção, dada a tecnologia disponível. A renda per capita é uma proxy da produtividade municipal e das condições tecnológicas em que está sendo realizada a produção agregada. Portanto, é possível assumir que a desigualdade de renda gerada pelas diferenças nas condições de produção afeta a formação de riquezas e o bem-estar dos agentes. A teoria neoclássica prevê que a renda per capita entre regiões tende a convergir para o mesmo estado estacionário devido aos retornos decrescentes do estoque capital (SOLOW, 1956, 1957). Porém, como as economias divergem em potencial de produção, elas podem convergir para diferentes estados estacionários (MANKIW et al., 1992). As diferenças entre equilíbrios representam desigualdades que podem se manter por longos períodos de tempo. Uma das explicações mais aceitáveis para as desigualdades econômicas persistentes é a erosão da capacidade de absorção de novas tecnologias. Essa interpretação sugere que existem limites de diferenças estruturais e tecnológicas cuja difusão de tecnologias se torna inócua sobre os diferenciais de renda entre economias ricas e pobres (QUAH, 1996; SCHIOPU, 2015). 
A convergência absoluta sugere uma aproximação da renda per capita entre regiões negligenciando suas especificidades. Quando estas são consideradas, a convergência passa a ser condicional. Até o início dos anos 2000, a evolução das desigualdades de renda entre os municípios brasileiros tinha como principais características a convergência para diferentes equilíbrios com persistência das desigualdades, formando diferentes grupos econômicos ou clubes de convergência (ANDRADE et al., 2002). Vários trabalhos apontam indícios de convergência condicional com fortes desequilíbrios regionais. Entre as interpretações sobre os resultados, considera-se como responsáveis pelas desigualdades: as diferenças tecnológicas, de conhecimento, de capital humano, de infraestrutura econômica e instituições (AZZONI et al., 2000; CHEIN; LEMOS; ASSUNÇÃO, 2007; CASALI; SILVA; CARVALHO, 2010; GONÇALVES; RIBEIRO; FREGUGLIA, 2011; BARROS, 2011; FIRME; FREGUGLIA, 2013).

Além das desigualdades regionais, a literatura empírica aponta, ainda, fortes desequilíbrios entre os estados e os municípios das regiões do país (RIBEIRO, 2010; CASALI; SILVA; CARVALHO, 2010; GONÇALVES; RIBEIRO; FREGUGLIA, 2011; FIRME; FREGUGLIA, 2013). Nesse contexto de análises empíricas, este artigo tem como objetivo incluir o efeito da inovação e difusão tecnológica na formação dos clubes de convergência dos municípios brasileiros. Para isso, são utilizados dois métodos: o modelo de misturas finitas (MMF) e a equação threshold, sob a alegação de baixa consistência dos estimadores de convergência em um único equilíbrio (DURLAUF; JOHNSON, 1995). O MMF usa os valores modais da distribuição dinâmica na determinação dos clubes de convergência econômica, agrupando os municípios por semelhança da distribuição de probabilidade dos determinantes de crescimento (OWEN; VIDERAS; DAVIS, 2009). A equação threshold estima os clubes de convergência a partir dos valores threshold do estoque de capital nos pontos não côncavos da função de produção agregada. A existência dos clubes com diferenças nas elasticidades dos fatores de produção e tecnológicos sugere que a função de produção agregada de Solow deve incorporar diferenças de produtividade (DURLAUF; JOHNSON, 1995; HANSEN, 1999, 2000, 2011).

Além desta introdução, o artigo se estrutura em mais três seções: a seção 2 apresenta o modelo empírico utilizado nos exercícios de convergência juntamente com suas respectivas variáveis e fontes dos dados; a seção 3 estima os clubes de convergência segundo os métodos de distribuição dinâmica (MMF) e equação threshold; e para finalizar, a seção 4 apresenta as considerações finais.

\section{Modelo Empírico, Apresentação das Variáveis e Fontes dos Dados}

O modelo de Solow parte de uma função de produção agregada com rendimentos constante de escala no longo prazo, que representa a agregação das 
funções de produção das firmas individuais. Contudo, a literatura aponta críticas em relação à agregação de funções de produção que representam tecnologias dissimilares. Isto é, a agregação de diferentes habilidades individuais do trabalho e a heterogeneidade das máquinas e equipamentos do estoque de capital (PRESSMAN, 2005). Por causa disso, existem alguns estudos que procuram mostrar que as estimativas derivadas de modelos, como o de Solow, podem não ser realistas (FELIPE; MCCOLOMBIE, 2005; FELIPE; FISHER, 2003).

Entretanto, por causa da necessidade de se estudar problemas econômicos agregados e pela adequação estatística dos parâmetros estimados, o modelo de Solow, ainda que com transformações, é largamente utilizado em trabalhos empíricos. Nas análises de convergência, o modelo neoclássico é utilizado e difundido em trabalhos empíricos seminais da teoria econômica aplicada como os de Mankiw et al. (1992) e Durlauf e Johnson (1995). Por essa razão, o modelo empírico de convergência municipal utilizado nesta pesquisa usa como referência o modelo neoclássico de crescimento (equação 1 a seguir), denominando-se $k_{j t}$ como o estoque de capital físico resultado dos investimentos das empresas, $g_{j t}$ como o estoque de capital dos investimentos do governo, e $h_{j t}$ como o estoque de capital humano.

$$
y_{j t}=k_{j t}^{\alpha} g_{j t}^{\theta} h_{j t}^{\delta} A_{j t}^{1-\alpha-\theta-\delta}, \operatorname{com} 0<\alpha, \theta, \delta<1
$$

O resíduo tecnológico da função de produção ampliada $\left(A_{j i}\right)$ é especificado para captar os efeitos da inovação e difusão tecnológica sobre o crescimento econômico e convergência de renda per capita municipal. Derivando-se o logaritmo da função de produção com spillovers tecnológicos em relação ao tempo, chega-se à equação empírica do crescimento econômico endógeno com capital humano, inovação e absorção de spillovers. Agrupando-se as variáveis explicativas em dois vetores, têm-se o vetor da dotação de fatores $(x)$, composto pelas variáveis de capital físico e humano, e o vetor tecnológico $(w)$, composto pelas variáveis de inovação e difusão tecnológica (ver Apêndice A):

$$
y=\beta x+\emptyset w+\mu_{j}
$$

A definição de variáveis e as fontes de informação estão apresentadas no Quadro 1. As variáveis são apresentadas em taxas de crescimento mediante a diferença dos logaritmos naturais entre os anos de 2000 e 2010, salvo o logaritmo da renda per capita inicial que por definição está em um ano fixo, e a qualidade institucional construída sob a hipótese de inércia institucional (BARROS NETO; NAKABASHI, 2011). A variável endógena nos dois modelos empíricos é a taxa de crescimento econômico da renda per capita municipal . A variável exógena que captura o parâ- 
metro de convergência econômica do modelo $\beta$-convergência é a renda per capita inicial, ou seja, a renda interna bruta per capita dos municípios em 2000.

Quadro 1 - Definição das variáveis e fontes dos dados

\begin{tabular}{|c|c|c|c|}
\hline Variáveis & Definição das variáveis do modelo $\beta$-convergência & Período & Fonte \\
\hline$\hat{y}_{j}$ & $\begin{array}{l}\text { Taxa de crescimento da renda per capita dos municípios j a } \\
\text { preços constantes de } 2000 \text {. }\end{array}$ & $2000-2010$ & $\begin{array}{c}\text { IBGE } \\
(2000,2010)\end{array}$ \\
\hline \multirow[t]{2}{*}{$\ln y_{j 0}$} & Renda per capita do município j em 2000. & 2000 & $\begin{array}{l}\text { IBGE } \\
(2000) \\
\text { IPEADATA } \\
(2000)\end{array}$ \\
\hline & Vetor da dotação de fatores & & \\
\hline$\hat{k}_{j}$ & $\begin{array}{l}\text { Taxa de crescimento da participação da renda industrial na } \\
\text { renda municipal a preços constantes de } 2000 .\end{array}$ & $2000-2010$ & $\begin{array}{c}\text { IBGE } \\
(2000,2010) \\
\end{array}$ \\
\hline$\hat{g}_{j}$ & $\begin{array}{c}\text { Taxa de crescimento dos gastos per capita em investimento dos } \\
\text { municípios j a preços constantes de } 2000 .\end{array}$ & $2000-2010$ & \begin{tabular}{|l|} 
IPEADATA \\
$(2000,2010)$ \\
\end{tabular} \\
\hline \multirow[t]{2}{*}{$\hat{h}_{j}$} & $\begin{array}{l}\text { Taxa de crescimento do número de pessoas de } 25 \text { anos ou } \\
\text { mais com o ensino fundamental concluído per capita. }\end{array}$ & $2000-2010$ & $\begin{array}{l}\text { IBGE } \\
(2000,2010) \\
\text { IPEADATA } \\
(2000,2010)\end{array}$ \\
\hline & Vetor de inovação e absorção da escalar tecnológica & & \\
\hline$g \hat{a} p_{j}$ & $\begin{array}{c}\text { Taxa de crescimento da diferença de produtividade industrial } \\
\text { entre a economia de maior produtividade industrial e as } \\
\text { demais. }\end{array}$ & $2000-2010$ & $\begin{array}{c}\text { IBGE } \\
(2000,2010) \\
\text { IPEADATA } \\
(2000,2010) \\
\end{array}$ \\
\hline$\hat{i}_{j}$ & $\begin{array}{l}\text { Taxa de crescimento da infraestrutura tecnológica composta } \\
\text { pelo componente principal das seguintes variáveis: número } \\
\text { de domicílios particulares com iluminação elétrica, com } \\
\text { microcomputadores, número de linhas telefônicas instaladas } \\
\text { ou de telefones móveis e número de domicilios com televisão. }\end{array}$ & $2000-2010$ & $\begin{array}{c}\text { IBGE } \\
(2000,2010)\end{array}$ \\
\hline$\hat{r}_{j}$ & $\begin{array}{l}\text { Taxa de crescimento do investimento em P\&D composto } \\
\text { pelo número de profissionais correlacionados com atividades } \\
\text { intensivas em P\&B em termos per capita. }\end{array}$ & $2000-2010$ & $\begin{array}{c}\text { RAIS } \\
(2000,2010)\end{array}$ \\
\hline$\hat{q}_{j}$ & $\begin{array}{l}\text { Logaritmo natural do índice de qualidade institucional dos } \\
\text { municípios. }\end{array}$ & 2000 & $\begin{array}{l}\text { MPLAN } \\
(2000)\end{array}$ \\
\hline$\hat{t}_{j}$ & $\begin{array}{l}\text { Taxa de crescimento das transferências tecnológicas composta } \\
\text { pelas importações de máquinas e equipamentos de alta e } \\
\text { média-alta intensidade tecnológica vindos dos países da } \\
\text { OCDE. }\end{array}$ & $2000-2010$ & $\begin{array}{c}\text { MDIC } \\
(2000,2010)\end{array}$ \\
\hline$\hat{l}_{j}$ & $\begin{array}{l}\text { Spillover de conhecimento composto pela interação entre a e } \\
\text { a população com ensino superior concluído em } 2000 .\end{array}$ & $2000-2010$ & $\begin{array}{l}\text { MDIC } \\
(2000,2010) \\
\text { IBGE } \\
(2000)\end{array}$ \\
\hline
\end{tabular}

Fonte: IBGE (2000, 2010); IPEADATA (2000, 2010); RAIS (2000, 2010); MPLAN (2000); MDIC $(2000,2010)$.

Nota: Todas as variáveis monetárias foram deflacionadas em relação aos preços de 2000. 
O vetor de variáveis explicativas relativas à dotação de fatores inclui:

a) a taxa de crescimento do capital físico municipal $\left(\hat{k}_{j}\right)$, medida pelo crescimento da participação do valor agregado industrial na renda interna municipal; ${ }^{1}$

b) o investimento público municipal per capita $\left(\hat{g}_{j}\right)$, medido como o crescimento do gasto municipal em investimento; ${ }^{2} \mathrm{e}$

c) o capital humano $\left(\hat{h}_{j}\right)$, medido pelo crescimento da participação da população maior de 25 anos com nível educacional fundamental concluído na população total do município.

As variáveis de inovação e absorção de tecnologia explicitadas através da escalar tecnológica da função de produção ampliada com capital humano são:

a) a taxa de infraestrutura tecnológica $\left(\hat{\imath}_{j}\right)$, medida pelo crescimento per capita do componente principal do número de domicílios permanentes com iluminação elétrica, microcomputador, linha telefônica instalada ou telefone celular e existência de televisão (CASTELLACI, 2011);

b) a taxa de investimento em pesquisa e desenvolvimento (P\&D) $\left(\hat{r}_{j}\right)$, medida como o crescimento do número de profissionais técnicos envolvidos em atividades de PEDD sobre a população municipal (ARAÚJO; CAVALCANTE; ALVES, 2009); ${ }^{3}$

c) o hiato de produtividade industrial, medido pela variável $g a \hat{a} p_{j}=\left[1-\frac{\hat{g}_{j}}{\hat{g}_{\text {Max }}}\right]$ ou seja, pela diferença entre as taxas de crescimento da produtividade industrial dos municípios $\left(\hat{g}_{j}\right)$ em relação ao crescimento máximo $\left(\hat{g}_{\text {Max }}\right)$ da razão entre produto e emprego industrial (RIBEIRO, 2010);

d) a taxa de crescimento da transferência de tecnologia incorporada importada $\left(\hat{t}_{j}\right)$, medida pelo crescimento da importação de produtos de médio e alto conteúdo tecnológico procedente de países da Organização

$1 \quad$ Uma proxy semelhante de investimento foi construída por Gonçalves, Ribeiro e Freguglia (2011) usando gasto público ao invés da renda industrial. Contudo, como a produção industrial é o resultado do investimento, entende-se que essa seria uma medida robusta da formação de capital municipal.

2 Conforme a Lei no. 4.320/64, o investimento público engloba dotações para o planejamento e execução de obras e aquisição de instalações, equipamentos e material permanente. Os dados sobre formação de capital público estão disponíveis no banco de dados da Secretaria do Tesouro Nacional do Ministério da Fazenda (STN).

3 A variável inclui pesquisadores, engenheiros, profissionais científicos, diretores e gerentes de P\&D. Foram selecionadas as seguintes categorias profissionais: químicos; físicos; engenheiros agrônomos, florestais e de pesca; engenheiros civis e arquitetos; engenheiros de operações, desenhistas industriais; engenheiros; profissionais da biotecnologia; profissionais da metrologia; engenheiros mecatrônicos; profissionais da matemática; profissionais de estatística; físicos; químicos; profissionais do espaço e da atmosfera; geólogos e geofísicos; engenheiros ambientais e afins; arquitetos; engenheiros civis e afins; engenheiros eletroeletrônicos e afins; engenheiros mecânicos; engenheiros químicos; engenheiros metalurgistas e de materiais; engenheiros de minas; engenheiros agrimensores e engenheiros cartógrafos; engenheiros industriais, de produção e segurança; biólogos e afins; biomédicos; engenheiros agrossilvipecuários; engenheiros de alimentos e afins. 
para a Cooperação e Desenvolvimento Econômico (OCDE) (FALVEY; FOSTER; GREENAWAY, 2007; RIBEIRO, 2010); ${ }^{4} \mathrm{e}$

e) os spillovers externos de tecnologias $\left(\hat{l}_{\hat{l}}\right)$, calculados como uma variável de interação entre a $\hat{t}_{j}$ e a população com ensino superior em 2000

$\left(\right.$ hj $_{2000}$ ) (FALVEY; FOSTER; GREENAWAY, 2007; COE; HELPMAN; HOFF MAISTER, 1997).

A variável que captura as diferenças de caráter institucional entre municípios não é introduzida nas estimações em taxas de crescimento, característica que se justifica devido à hipótese de inércia institucional (BARROS NETO; NAKABASHI, 2011). ${ }^{5}$ A qualidade institucional $\left(\mathrm{q}_{\mathrm{j}}\right)$ é medida através do logaritmo natural do índice de qualidade institucional municipal (ver Apêndice B). A qualidade institucional é disponibilizada pelo Ministério do Planejamento, Orçamento e Gestão (MPOG) apenas para o ano de 2000.

\section{Estimativa dos Clubes de Convergência Municipal entre 2000 e 2010}

A análise dos clubes de convergência é originalmente apresentada por Quah (1996) para identificar a persistência de múltiplos picos modais em funções temporais de distribuição de probabilidade da renda per capita das economias. Neste trabalho, os clubes de convergência e as elasticidades da dotação de fatores e difusão tecnológica são estimados utilizando-se o MMF e a equação threshold.

Por construção matemática, a $\beta$-convergência é o parâmetro da renda per capita inicial $\left(y_{i 0}\right)$ que explica a taxa de crescimento da renda per capita $\left(\frac{1}{T} \ln \left(\frac{y_{i t}}{y_{i 0}}\right)=\beta_{0}+\left(\frac{1-e^{-\lambda t}}{T}\right) \ln y_{i 0}\right)$ (BARRO; SALA-I-MARTIN, 1992). O parâmetro $\beta$-convergência é dado pelo coeficiente $\left(\frac{1-e^{-\lambda t}}{T}\right)$, que inclui um componente de tempo para mostrar a redução do valor do parâmetro à medida que as economias vão se aproximando ao estado estacionário. No longo prazo, o valor do parâmetro de convergência diminui, refletindo a redução das diferenças entre as taxas de crescimento cross section.

O MMF estima os clubes pela semelhança da distribuição de probabilidade da taxa de crescimento econômico condicionada aos determinantes imediatos do crescimento econômico. O MMF é uma regressão de classe latente que pode ser empregado para estimar as distribuições discretas dos regimes de crescimento econômico, e é apresentado pela teoria dos regimes de equilíbrios múltiplos como o método

$4 \quad$ Os produtos de médio e médio-alto conteúdo tecnológico definidos com base em ISIC (2011).

5 A mudança institucional possui um caráter inercial devido à persistência das desigualdades institucionais (ACEMOGLU; JOHNSON; ROBINSON, 2001). 
mais apropriado para o teste de convergência em clubes, pois identifica a fonte da heterogeneidade sistemática das economias (OWEN; VIDERAS; DAVIS, 2009).

O método threshold estima a função de produção agregada dos municipios brasileiros baseado na teoria dos regimes de equilibrios múltiplos. Nessa teoria, a função do investimento per capita (s $f(k))$ é decrescente, salvo as exceções dos pontos de capital per capita threshold, nos quais a função de produção não é côncava. A função de depreciação $((n+g+\delta) k)$ mantém sua linearidade independente do retorno decrescente dos novos investimentos (ver Gráfico 1). A teoria dos equilíbrios múltiplos assume que as economias em cada regime de crescimento estão submetidas aos retornos decrescentes do estoque de capital. Portanto, cada regime está convergindo para o seu próprio estado estacionário, formando clubes de convergência. A diferença entre economias avançados e atrasadas é dada por um nível threshold do estoque de capital $\left(k^{\mathrm{T}}\right)$ não observado, implicando diferenças de produtividade na função de produção agregada de Solow (DURLAUF; JONSHON, 1995).

Gráfico 1 - Regime econômico de crescimento com múltiplos equilíbrios

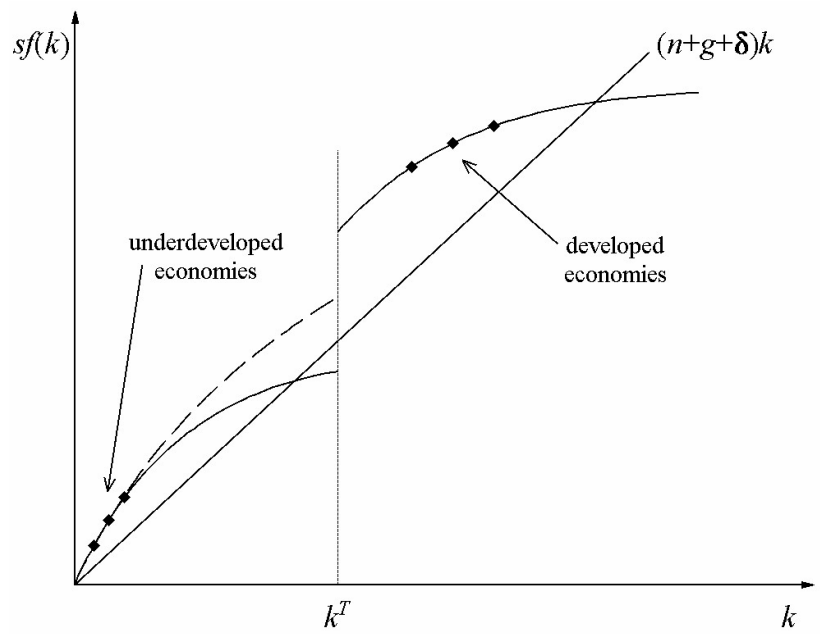

Fonte: Durlauf, Johnson e Temple (2004, p. 96).

\subsection{Estimativa dos Clubes de Convergência por Distribuição Dinâmica}

A distribuição dinâmica dos clubes convergência é comumente medida através da função kernel de densidade de probabilidade, que consiste numa generalização do histograma dado por uma função alternativa de pesos na qual é 
a função kernel, $N$ é o número de observações e $h$ é o parâmetro de alisamento (CATELA; GONÇALVES, 2009).

$$
\hat{f}\left(x_{0}\right)=\frac{1}{N h} \sum_{i=1}^{N} k\left(\frac{x_{i}-x_{0}}{h}\right)
$$

As funções de densidade ilustram as análises exploratórias de dados segundo os aspectos de multimodalidade, assimetria e curtose. A referida análise é largamente utilizada na caraterização de variáveis devido a sua simplicidade, ausência de viés e consistência. A função kernel é uma estatística não paramétrica, portanto não depende do conhecimento a priori da distribuição dos dados (LUCAMBIO, 2008). O uso do logaritmo nas variáveis da distribuição de probabilidade deve ser utilizado para corrigir distorções e outliers dos dados (BIANCHI, 1997).

A formação dos picos modais na distribuição de probabilidade da renda per capita dos municípios brasileiros é consistente com a hipótese de clubes de convergência. Ou seja, significa que os referidos municípios estão numa trajetória de crescimento equilibrado com a formação de dois clubes de convergência (ver Gráfico 2). Como pressupõe a teoria dos clubes convergência em "twin peaks", o equilíbrio de longo prazo acontece com a formação de um clube subdesenvolvido e outro desenvolvido. Contudo, esse resultado constitui uma evidência pouco explicativa a priori sobre a trajetória de convergência em clube dos municípios brasileiros.

Gráfico 2 - Densidade kernel do logaritmo da renda per capita municipal em 2000 e 2010

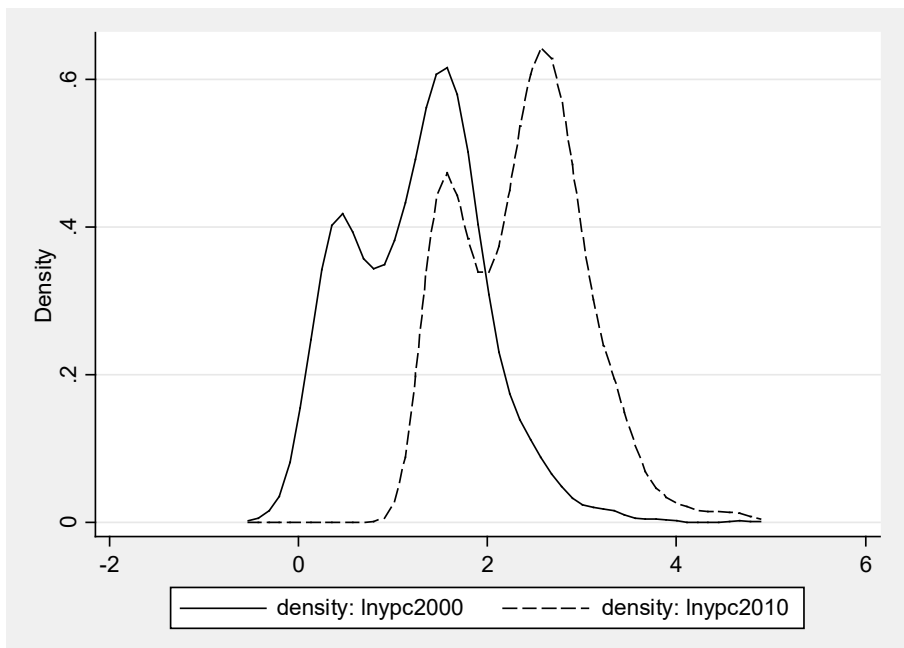

Fonte: Elaboração própria a partir de dados do IBGE (2000, 2010) 
A formação de dois clubes de convergência dos municípios brasileiros em 2000 e 2010 retrata a persistência das desigualdades municipais no tempo. Entre 1970 e 1996, já havia sido constatado um clube de baixa renda, constituído pelos municípios do Norte e Nordeste, e outro de alta renda, formado pelos municípios do Sul, Sudeste e Centro-Oeste do país (LAURINI; ANDRADE; PEREIRA, 2003). Apesar disso, não se pode generalizar que as desigualdades de renda se devam unicamente aos fatores regionais. Existem municípios de alto desenvolvimento em regiões pobres, assim como municípios pobres em regiões ricas. Nas regiões Norte e Nordeste, existem municípios de grande produtividade, ao mesmo tempo que, nas regiões Sul e Sudeste, existem municípios de baixa produtividade (CHEIN; LEMOS; ASSUNÇÃO, 2007; CATELA; GONÇALVES, 2009).

\subsection{Clubes de Convergência Estimados por Misturas Finitas}

O estimador MMF equaciona a multimodalidade da distribuição dinâmica sem ordenar os clubes. Portanto, é difícil afirmar a situação de desenvolvimento em que se encontram os clubes estimados. Para isso, deve-se estimar a convergência dos municípios em clubes, a qual identifica as elasticidades dos determinantes de crescimento em cada um dos clubes de convergência. Como a função kernel exibe dois valores modais da renda per capita, espera-se que a equação por MMF mais consistente tenha também dois componentes (CAMERON; TRIVEDI, 2005). A principal característica desse modelo é a estimação simultânea dos parâmetros de elasticidade por clubes de convergência econômica.

O MMF supõe que a densidade de $y$ é uma combinação linear de $m$ diferentes densidades dadas por $f\left(y / \theta_{j}\right) \operatorname{com} j=1, \ldots, m$ densidades. Os componentes estimados capturam os valores modais da função densidade de probabilidade, agrupando a população de dados em subpopulações $\left(\pi_{j}\right)$, denominadas clubes de convergência (CAMERON; TRIVEDI, 2005). A equação empírica estimada a partir da equação genérica do $\mathrm{MMF}$, em que $\sum_{j=1}^{m} \pi_{j}=1$, é a seguinte:

$$
f(y / \theta, \pi)=\sum_{j=1}^{m} \pi_{j} f_{j}\left(y / y_{0 j}, x_{j}, w_{j}, d_{n-1}\right)+\varepsilon_{i}
$$

na qual y é a variável dependente de crescimento da renda municipal per capita de 2000 a 2010, condicionada aos vetores de variáveis explicativas $y_{0 j}, x_{j}$, $w_{j}, d_{n-1}$, dos quais $y_{0_{j}}$ é o logaritmo natural da renda per capita no ano de 2000. O vetor de variáveis $x_{j}$ refere-se ao crescimento do capital humano per capita $(\hat{h})$, ao crescimento da taxa de industrialização $\left(\hat{k}_{j}\right)$ e ao crescimento do investimento público per capita $\left(\hat{g}_{j}\right)$. O vetor de variáveis $w_{j}$ corresponde ao logaritmo natural da qualidade institucional dos municípios $\left(\hat{q}_{j}\right)$, ao crescimento dos gastos 
em infraestrutura tecnológica $(\hat{i})$, ao crescimento do investimento em P\&D $\left(\hat{r}_{j}\right)$, ao crescimento do gap tecnológico ( $g \hat{a} p_{j}$ ), ao crescimento da importação de tecnologias de médio e alto conteúdo tecnológico $\left(\hat{t}_{j}\right)$ e ao crescimento dos spillovers de conhecimento por interação $(\hat{l})$. O vetor $d_{n-1}$ representa as $n-1$ regiões do país.

Os resultados estimados por MMF para os municípios demonstram a existência de dois clubes de convergência (ver Tabela 1). Embora tenham sido realizados os exercícios com três componentes, ou seja, simulando três clubes de convergência, a equação com dois componentes exibe maior consistência em relação à equação com três componentes. Tanto na equação de dois quanto na de três componentes, os parâmetros estimados apresentam maior consistência em relação ao modelo de equilíbrio único estimado por mínimos quadrados ordinários (MQO). Em condições de regularidade, a estimativa de regressão por partes converge para um melhor preditor não linear da variável dependente de interesse (BREIMAN et al., 1984). Baseado nisso, Durlauf e Johnson (1995) apresentam o método de regressões em árvore na estimativa de convergência em múltiplos equilíbrios como mais consistente que o método de equilíbrio único.

Tabela 1 - Clubes de convergência estimados por mínimos quadrados ordinários e misturas finitas

\begin{tabular}{|c|c|c|c|c|c|c|}
\hline \multirow[t]{2}{*}{ Variáveis } & \multirow{3}{*}{$\begin{array}{c}\text { MQO } \\
-0.160^{* * *} \\
(-14.1)\end{array}$} & \multicolumn{2}{|c|}{$\begin{array}{l}\text { Regressão com dois } \\
\text { componentes }\end{array}$} & \multicolumn{3}{|c|}{ Regressão com três componentes } \\
\hline & & $-0.191 * * *$ & $-0.177 * * *$ & $-0.079 * * *$ & $-0.1904 * * *$ & $-0.227 * * *$ \\
\hline $\ln y_{0 j}$ & & $(-16.2)$ & $(-3.74)$ & $(-4.01)$ & $(2.83)$ & $(-13.3)$ \\
\hline$\hat{k}_{j}$ & $\begin{array}{c}0.0742^{* * * *} \\
(4.16)\end{array}$ & $\begin{array}{c}0.0514^{* * *} \\
(2.96)\end{array}$ & $\begin{array}{c}0.1190^{*} \\
(1.93)\end{array}$ & $\begin{array}{c}-0.317 * * * \\
(-5.07)\end{array}$ & $\begin{array}{c}0.1708 * * \\
(2.36)\end{array}$ & $\begin{array}{c}0.1943 * * * \\
(6.69)\end{array}$ \\
\hline$\hat{g}_{j}$ & $\begin{array}{c}0.0149 * * * \\
(3.79)\end{array}$ & $\begin{array}{c}0.0074^{* * *} \\
(2.37)\end{array}$ & $\begin{array}{c}0.0563^{* *} \\
(2.20)\end{array}$ & $\begin{array}{c}0.0003 \\
(0.05)\end{array}$ & $\begin{array}{c}0.0607^{* *} \\
(1.98)\end{array}$ & $\begin{array}{c}0.0143^{* * *} \\
(3.02)\end{array}$ \\
\hline$\hat{\imath}$ & $\begin{array}{c}0.0401^{* * *} \\
(2.77)\end{array}$ & $\begin{array}{c}0.0448^{* * *} \\
(3.48)\end{array}$ & $\begin{array}{l}-0.0734 \\
(-0.84)\end{array}$ & $\begin{array}{c}0.0392 \\
(1.30)\end{array}$ & $\begin{array}{c}-0.0362 \\
(-0.33)\end{array}$ & $\begin{array}{c}0.0377^{* *} \\
(2.07)\end{array}$ \\
\hline$g \hat{a} p_{j}$ & $\begin{array}{c}0.0690 * * * \\
\quad(3.01)\end{array}$ & $\begin{array}{c}0.0567^{* * *} \\
\quad(3.06)\end{array}$ & $\begin{array}{c}0.0813 \\
(0.66)\end{array}$ & $\begin{array}{c}0.0415 \\
(1.16)\end{array}$ & $\begin{array}{c}0.0830 \\
(0.60)\end{array}$ & $\begin{array}{c}0.0679 * * \\
(2.55)\end{array}$ \\
\hline$\hat{i}_{j}$ & $\begin{array}{c}0.1342 * * * \\
(14.72)\end{array}$ & $\begin{array}{c}0.1085 * * * \\
(13.02)\end{array}$ & $\begin{array}{c}0.2288^{* * *} \\
\quad(5.08)\end{array}$ & $\begin{array}{c}0.0527^{* * *} \\
(2.62)\end{array}$ & $\begin{array}{c}0.2704^{* * *} \\
\quad(5.61)\end{array}$ & $\begin{array}{c}0.1326 * * * \\
(11.11)\end{array}$ \\
\hline$\hat{r}_{j}$ & $\begin{array}{c}0.0169^{* * * *} \\
(2.89)\end{array}$ & $\begin{array}{c}0.0119^{*} \\
(1.94)\end{array}$ & $\begin{array}{c}0.0419 * * \\
(2.16)\end{array}$ & $\begin{array}{c}0.0008 \\
(0.11)\end{array}$ & $\begin{array}{c}0.0533^{* *} \\
(2.04)\end{array}$ & $\begin{array}{c}0.0230 * * * \\
(2.65)\end{array}$ \\
\hline$\hat{q}_{j}$ & $\begin{array}{c}0.1179 * * * \\
(4.39)\end{array}$ & $\begin{array}{c}0.0887^{* * *} \\
(3.47)\end{array}$ & $\begin{array}{c}0.3533^{* *} \\
(2.51)\end{array}$ & $\begin{array}{c}0.0636 \\
(1.17)\end{array}$ & $\begin{array}{c}0.4404^{* * *} \\
\quad(2.65)\end{array}$ & $\begin{array}{c}0.0867^{* * *} \\
(2.51)\end{array}$ \\
\hline$\hat{t}_{j}$ & $\begin{array}{c}0.0079^{* * *} \\
\quad(4.74)\end{array}$ & $\begin{array}{c}0.0035^{* * *} \\
(3.04)\end{array}$ & $\begin{array}{c}0.0246 * * * \\
\quad(3.25)\end{array}$ & $\begin{array}{c}0.0042 \\
(0.99)\end{array}$ & $\begin{array}{c}0.0276^{* * *} \\
(3.33)\end{array}$ & $\begin{array}{c}0.0024 \\
(1.37)\end{array}$ \\
\hline$\hat{l}_{j}$ & $\begin{array}{c}8.8 \mathrm{E}-07 * * * \\
(2.69)\end{array}$ & $\begin{array}{c}1.15 \mathrm{E}-6^{* * *} \\
(3.23)\end{array}$ & $\begin{array}{c}3.1 \mathrm{E}-07 \\
(0.12)\end{array}$ & $\begin{array}{c}5.91 \mathrm{E}-08 \\
(0.28)\end{array}$ & $\begin{array}{c}-2.34 \mathrm{E}-07 \\
(0.22)\end{array}$ & $\begin{array}{c}3.29 \mathrm{E}-06^{* * *} \\
(2.37)\end{array}$ \\
\hline
\end{tabular}

continua... 
conclusão.

\begin{tabular}{|c|c|c|c|c|c|c|}
\hline \multirow{2}{*}{$\begin{array}{l}\text { Variáveis } \\
\text { Norte }\end{array}$} & \multirow{2}{*}{$\begin{array}{c}\text { MQO } \\
0.1084^{* * *} \\
(5.40)\end{array}$} & \multicolumn{2}{|c|}{$\begin{array}{l}\text { Regressão com dois } \\
\text { componentes }\end{array}$} & \multicolumn{3}{|c|}{ Regressão com três componentes } \\
\hline & & $\begin{array}{c}0.0907^{* * *} \\
(3.85)\end{array}$ & $\begin{array}{c}0.1830 * * \\
(2.27)\end{array}$ & $\begin{array}{c}0.0807^{*} \\
(1.69)\end{array}$ & $\begin{array}{c}0.2185^{* * *} \\
\quad(2.61)\end{array}$ & $\begin{array}{c}0.0839 * * \\
(2.38)\end{array}$ \\
\hline Sudeste & $\begin{array}{c}0.0702^{* * *} \\
\quad(5.13)\end{array}$ & $\begin{array}{c}0.0665^{* * *} \\
\quad(5.55)\end{array}$ & $\begin{array}{c}0.1981 * * \\
(2.41)\end{array}$ & $\begin{array}{c}-0.085^{* * *} \\
(-2.62)\end{array}$ & $\begin{array}{c}0.2632^{* *} \\
(2.56)\end{array}$ & $\begin{array}{c}0.1159 * * * \\
\quad(7.04)\end{array}$ \\
\hline Sul & $\begin{array}{c}0.1088 * * * \\
\quad(6.67)\end{array}$ & $\begin{array}{c}0.1708^{* * *} \\
(11.63)\end{array}$ & $\begin{array}{c}-0.0664 \\
(-0.85)\end{array}$ & $\begin{array}{c}0.0263 \\
(0.63)\end{array}$ & $\begin{array}{c}-0.0748 \\
(-0.78)\end{array}$ & $\begin{array}{c}0.2092^{* * *} \\
\quad(10.88)\end{array}$ \\
\hline $\begin{array}{l}\text { Centro- } \\
\text { Oeste }\end{array}$ & $\begin{array}{c}0.1246^{* * *} \\
(7.14)\end{array}$ & $\begin{array}{c}0.1724^{* * *} \\
(10.69)\end{array}$ & $\begin{array}{c}0.0020 \\
(0.02)\end{array}$ & $\begin{array}{c}0.0342 \\
(0.91)\end{array}$ & $\begin{array}{l}0.0105 \\
(0.11)\end{array}$ & $\begin{array}{c}0.2138 * * \\
(9.17)\end{array}$ \\
\hline Constante & $\begin{array}{c}1.0367^{* * *} \\
(26.01)\end{array}$ & $\begin{array}{c}1.0686^{* * *} \\
(31.64) \\
\end{array}$ & $\begin{array}{c}0.9694^{* * *} \\
(4.97)\end{array}$ & $\begin{array}{c}0.8660^{* * *} \\
(10.31)\end{array}$ & $\begin{array}{c}0.8945^{* * *} \\
(4.07)\end{array}$ & $\begin{array}{c}1.1498^{* * * *} \\
(22.91)\end{array}$ \\
\hline N. obs. & 4008 & 3697 & 311 & 708 & 240 & 3060 \\
\hline AIC & 281.9899 & \multicolumn{2}{|c|}{-640.090} & \multicolumn{3}{|c|}{-850.413} \\
\hline BIC & 376.4306 & \multicolumn{2}{|c|}{-432.320} & \multicolumn{3}{|c|}{-535.611} \\
\hline Entropia & & 0.567 & & 0.368 & & \\
\hline
\end{tabular}

Fonte: Elaboração própria a partir de dados do IBGE (200, 2010), IPEADATA (2000, 2010), RAIS (2000, 2010), MPLAN (2000), e MDIC $(2000,2010)$. Nota: *** significativo a $1 \%$, ** significativo a $5 \%,{ }^{*}$ significativo a $10 \%$; entre parênteses, as medidas de estatística $t$ de Student.

A equação com dois componentes exibe o maior coeficiente de entropia $(0,567)$, indicando maior ajuste em relação à equação de três componentes $(0,368)$. Quanto mais próximo do valor um, maior é a consistência dos componentes estimados. A equação com três componentes apresenta os dois últimos clubes com valores $\beta$-convergência semelhantes aos da equação com dois componentes. Esse resultado demonstra a representatividade dos clubes da equação de dois componentes. O caso que exibe um padrão de convergência menos acentuado na equação com três componentes pode estar refletindo um conjunto de outliers (CATELA, 2009).

O estimador MMF exibe clubes de convergência nos quais o efeito da dotação de fatores e o vetor tecnológico são maiores. Portanto, esses grupos de convergência são mais homogêneos e consistentes (DURLAUF; JOHNSON, 1995). O viés do estimador pela média é fortemente influenciado pela discrepância dos grupos de fraca convergência, afetando a consistência do estimador. A convergência econômica para um equilíbrio único se mostra menos robusta quando comparada aos clubes estimados.

A Tabela 2 apresenta o nível de separação entre os componentes, assumindo que a probabilidade média posterior deve ser alta nos clubes pertencentes à classe latente (CL) e baixa nas demais. A diagonal principal da Tabela 2 expressa a probabilidade por classe latente e deve ser maior do que nos demais componentes da matriz. A probabilidade média posterior das classes latentes da regressão com dois componentes é maior que na regressão com três componentes. As probabilidades 
fora da classe latente são menores para a equação com dois componentes. Esses dois resultados fortalecem a regressão com dois componentes em relação a outra.

Tabela 2 - Probabilidade média posterior das classes latentes estimadas por MMF

\begin{tabular}{l|cc||ccc}
\hline \hline \multirow{2}{*}{ Média } & \multicolumn{2}{|c||}{ Regressão com dois componentes } & \multicolumn{3}{c}{ Regressão com três componentes } \\
\cline { 2 - 5 } & CL1 & CL2 & CL1 & CL2 & CL3 \\
\hline P1 & 0.901 & 0.099 & 0.650 & 0.109 & 0.240 \\
P2 & 0.151 & 0.849 & 0.077 & 0.842 & 0.081 \\
P3 & & & 0.252 & 0.064 & 0.685 \\
\hline \hline
\end{tabular}

Fonte: Elaboração própria a partir de dados do IBGE (200, 2010); IPEADATA (2000, 2010); RAIS (2000, 2010); MPLAN (2000); MDIC (2000, 2010).

A equação com dois componentes é a mais ajustada para representar os múltiplos equilíbrios dos municípios brasileiros. De acordo com ela, existe um efeito significativo da dotação de fatores municipais sobre o crescimento, assim como os determinantes de difusão tecnológica nos dois clubes de convergência. De forma semelhante ao modelo de equilíbrio único estimado por $\mathrm{MQO}$, a convergência por clube demonstra que a maioria das variáveis da dotação de fatores e da difusão tecnológica são significativas para explicar o crescimento econômico municipal. A significância estatística das dummies regionais nos dois modelos indica que os municípios do Nordeste são os que menos crescem em relação as demais regiões.

Embora o MMF seja recomendado para estimar regressões de classe latente compatíveis com o teste de convergência em clubes, a ausência de ordenação dos clubes é uma limitação inerente ao modelo. Sua principal característica é estimar parâmetros de elasticidades distintas em cada um dos grupos econômicos identificados. Portanto, seu uso se restringe ao cálculo das elasticidades das variáveis correspondentes à dotação de fatores e difusão tecnológica dos clubes de convergência visualizados na distribuição bimodal do logaritmo da renda per capita dos municípios brasileiros.

\subsection{Estimativa Threshold dos Clubes de Convergência}

A regressão threshold é um estimador não linear com especificações lineares por partes. A não linearidade dos parâmetros estimados é determinada nos momentos em que a variável observada cruza os valores threshold do estoque de capital a priori desconhecidos. A literatura sugere que esses valores threshold sejam determinados através dos valores iniciais da renda e da taxa de educação (DURLAUF; JOHNSON, 1995; HANSEN, 2000). Os valores threshold agregados são identificados ao longo da função de produção mediante o teste quebra estrutural (BAI; PERRON, 1998). A escolha da variável threshold se dá através do critério de 
ajuste Akaike. Entre as variáveis candidatas a threshold, é escolhida a que possuir o menor valor do critério Akaike.

Desse modo, o crescimento econômico é condicionado a um vetor de variáveis $(X)$ com parâmetros específicos por regimes de crescimento econômico. Os parâmetros estimados mudam de acordo com o limiar $\gamma$ da variável threshold ( $q$ ) com $m+1$ regimes de crescimento (HANSEN, 2000):

$$
y=\mu_{j}+\beta_{1} x_{\mathrm{j}} I\left(q_{\mathrm{j}} \leq \gamma\right) \beta_{2} x_{\mathrm{j}} I\left(q_{\mathrm{j}}>\gamma\right)+\varepsilon_{\mathrm{j}}
$$

em que:

$$
y_{j}=\left\{\begin{array}{cc}
\mu_{j}+\hat{\beta_{1}^{\prime}} x_{j}+\varepsilon_{\mathrm{j}}, & q_{j} \leq \gamma \\
\mu_{j}+\grave{\beta_{2}} x_{j}+\varepsilon_{\mathrm{j}}, & q_{j}>\gamma
\end{array}\right.
$$

Decompondo-se o vetor $X$ em subvetores $x$ da dotação de fatores com renda inicial, $w$ das variáveis tecnológicas e um último de dummies de controle regional , tem-se a equação empírica do modelo:

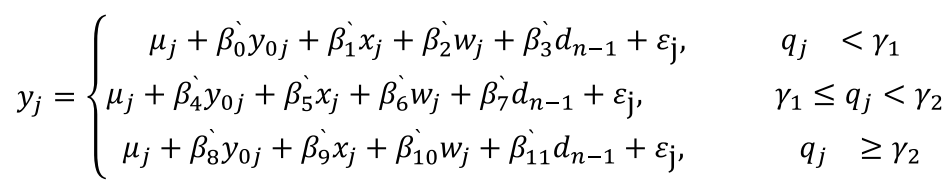

O estimador threshold dos clubes municipais de convergência demonstra de maneira geral que os efeitos da dotação de fatores e da difusão tecnológica são maiores comparativamente ao estimador linear de equilíbrio único, em ao menos um dos regimes estimados (ver Tabela 3). Esse resultado mostra que a equação threshold é mais consistente na análise de convergência com múltiplos equilíbrios, ou seja, o modelo de crescimento pode ser melhorado acrescentando diferenças de produtividade à função de produção agregada (DURLAUF; JOHNSON, 1995; HANSEN, 2000). Em uma economia com múltiplos equilíbrios, o estimador MQO apresenta necessariamente resultados não consistentes e muito vulneráveis a informações discrepantes. 
Tabela 3 - Estimador threshold dos clubes de convergência

\begin{tabular}{|c|c|c|c|c|}
\hline \multirow{2}{*}{ Variáveis } & Coeficientes & Regime 1 & Regime 2 & Regime 3 \\
\hline & MQO & Threshold $<\gamma_{1}$ & $\gamma_{1} \leq$ Threshold $<\gamma_{2}$ & Threshold $\geq \gamma_{2}$ \\
\hline $\mathrm{h} y_{0 j}$ & $\begin{array}{c}-0.1600 * * * \\
(-13.48)\end{array}$ & $\begin{array}{c}-0.2892 * * * \\
(-7.5961)\end{array}$ & $\begin{array}{c}-0.1431 * * * \\
(-7.6969)\end{array}$ & $\begin{array}{c}-0.1601 * * * \\
(-8.1732)\end{array}$ \\
\hline$\hat{k}_{j}$ & $\begin{array}{c}0.0742 * * * \\
(4.035)\end{array}$ & $\begin{array}{c}-0.0085 \\
(-0.2518)\end{array}$ & $\begin{array}{l}0.0942 * * * \\
(3.94055)\end{array}$ & $\begin{array}{c}0.1104^{* * *} \\
(3.1705)\end{array}$ \\
\hline$\hat{g}_{j}$ & $\begin{array}{c}0.0149 * * * \\
(3.8408)\end{array}$ & $\begin{array}{l}0.0112 * \\
(1.7831)\end{array}$ & $\begin{array}{c}0.0059 \\
(1.0003)\end{array}$ & $\begin{array}{c}0.0308 * * * \\
(4.0971)\end{array}$ \\
\hline$\hat{h}_{j}$ & $\begin{array}{c}0.0401^{* * *} \\
(2.6926)\end{array}$ & $\begin{array}{c}0.0696 * * * \\
(3.0671)\end{array}$ & $\begin{array}{c}0.0888 * * * \\
(2.8739)\end{array}$ & $\begin{array}{c}0.0310 \\
(0.8326)\end{array}$ \\
\hline$g \hat{a} p_{j}$ & $\begin{array}{c}0.0690 * * * \\
(2.9086)\end{array}$ & $\begin{array}{c}0.0383 \\
(0.9846)\end{array}$ & $\begin{array}{l}0.0621 \% * \\
(1.9767)\end{array}$ & $\begin{array}{l}0.1072 * \\
(1.8829)\end{array}$ \\
\hline$\hat{i}_{j}$ & $\begin{array}{l}0.1342 * * * \\
(13.613)\end{array}$ & $\begin{array}{c}0.1203 * * * \\
(9.2698)\end{array}$ & $\begin{array}{c}0.1352 * * * \\
(8.6718)\end{array}$ & $\begin{array}{c}0.1689 * * * \\
(7.6459)\end{array}$ \\
\hline$\hat{r}_{j}$ & $\begin{array}{c}0.0169 * * * \\
(2.9885)\end{array}$ & $\begin{array}{l}0.0257^{*} \\
(1.8051)\end{array}$ & $\begin{array}{l}0.0196 * \\
(1.9078)\end{array}$ & $\begin{array}{l}0.0135^{*} \\
(1.7355)\end{array}$ \\
\hline$\hat{q}_{j}$ & $\begin{array}{c}0.1179 * * * \\
(4.3609)\end{array}$ & $\begin{array}{c}0.0236 \\
(0.5378)\end{array}$ & $\begin{array}{c}0.1164 * * * \\
(2.7637)\end{array}$ & $\begin{array}{c}0.1599 * * * \\
(2.8834)\end{array}$ \\
\hline$\hat{t}_{j}$ & $\begin{array}{c}0.0079 * * * \\
(4.8650)\end{array}$ & $\begin{array}{c}-0.0137 \\
(-0.6376)\end{array}$ & $\begin{array}{c}0.0133 * * * \\
(3.6760)\end{array}$ & $\begin{array}{c}0.0048 * * * \\
(2.9064)\end{array}$ \\
\hline$\hat{l}_{j}$ & $\begin{array}{c}8.8 \mathrm{E}-07 * * * \\
(2.6000)\end{array}$ & $\begin{array}{c}0.0003 * * * \\
(5.1366)\end{array}$ & $\begin{array}{c}6.52 \mathrm{E}-06 \\
(1.1778)\end{array}$ & $\begin{array}{c}6.75 \mathrm{E}-07^{* *} \\
(2.1998)\end{array}$ \\
\hline Norte & $\begin{array}{c}0.1084 * * * \\
(3.759)\end{array}$ & $\begin{array}{c}0.1915 * * * \\
(4.1979)\end{array}$ & $\begin{array}{c}0.0461 \\
(1.3647)\end{array}$ & $\begin{array}{c}0.0622 \\
(1.2730)\end{array}$ \\
\hline Sudeste & $\begin{array}{c}0.0702 * * * \\
(4.088)\end{array}$ & $\begin{array}{c}0.0948 * * * \\
(3.3848)\end{array}$ & $\begin{array}{c}0.0351 \\
(1.6434)\end{array}$ & $\begin{array}{c}-0.0022 \\
(-0.0712)\end{array}$ \\
\hline Sul & $\begin{array}{c}0.1088 * * * \\
(5.8395)\end{array}$ & $\begin{array}{c}0.2371 * * * \\
(4.8172)\end{array}$ & $\begin{array}{c}0.0987 * * * \\
(4.0909)\end{array}$ & $\begin{array}{c}0.0022 \\
(0.0703)\end{array}$ \\
\hline Centro-Oeste & $\begin{array}{c}0.1246 * * * \\
(6.3591)\end{array}$ & $\begin{array}{c}0.3751^{* * *} \\
(7.0205)\end{array}$ & $\begin{array}{c}0.1036 * * * \\
(4.2865)\end{array}$ & $\begin{array}{c}-0.0093 \\
(-0.2675)\end{array}$ \\
\hline Constante & $\begin{array}{c}1.0367^{* * * *} \\
(25.023)\end{array}$ & $\begin{array}{l}1.1147^{* * *} \\
(17.2089)\end{array}$ & $\begin{array}{l}1.0477 * * * \\
(17.0408)\end{array}$ & $\begin{array}{l}1.0522 * * * \\
(10.9567)\end{array}$ \\
\hline $\begin{array}{l}\text { Número de } \\
\text { observações }\end{array}$ & 4008 & 946 & 1515 & 1547 \\
\hline$R^{2}$ & & & 0.2 & \\
\hline AIC & & & 0.0 & \\
\hline $\operatorname{Pr}$ (F-estatistic) & & & 0.0 & \\
\hline
\end{tabular}

Fonte: Elaboração própria a partir dos dados do IBGE (200, 2010), IPEADATA (2000, 2010), RAIS (2000, 2010), MPLAN (2000), e MDIC (2000, 2010).

Nota: ${ }^{* * *}$ significativo a $1 \%,{ }^{* *}$ significativo a $5 \%,{ }^{*}$ significativo a $10 \%$; entre parênteses, as estatísticas do teste $t$ de Student. 
O estimador threshold da função de produção agregada identifica três regimes de crescimento ou clubes de convergência em múltiplos equilíbrios. Os referidos regimes que diferem em produtividade agregada são separados por valores threshod do estoque inicial de capital, cuja proxy é a taxa de ensino fundamental em 2000. ${ }^{6}$ Esse resultado mostra que os municípios com as maiores taxas iniciais de educação fundamental têm maior produtividade em relação aos municípios de educação mais atrasada. Assim como no modelo MQO, todos os determinantes do crescimento possuem significância estatística, especialmente nos regimes de crescimento de maior desenvolvimento.

No regime um, de menor nível de desenvolvimento, é identificada a maior velocidade de convergência com um valor $\beta$ de -0,2892, o qual mostra a homogeneidade da convergência entre os municípios mais pobres. Não é observada significância estatística da taxa de industrialização $(k)$. Mesmo assim, o capital humano $(h)$ explica o crescimento econômico dos municípios mais pobres a $1 \%$ de significância e os investimentos públicos $(g)$ a 10\%. A falta de significância da taxa de industrialização e da qualidade institucional $(q)$ no grupo municipal mais pobre pode estar associada à precariedade institucional desses municípios e a baixa produtividade dos fatores. Do ponto de vista institucional, entende-se que as desigualdades econômicas são causadas por desigualdades institucionais persistentes, que se consolidam em função do nível de desenvolvimento regional (ACEMOGLU; JOHNSON; ROBINSON, 2001). A ausência de efeito da taxa de industrialização no crescimento do grupo municipal atrasado é coerente com o dado de concentração da infraestrutura produtiva no centro econômico do país. Essa concentração exerce um efeito direto sobre a competitividade e o nível de investimento regional do Brasil (SCHETTINI; AZZONI, 2015, 2018).

O regime dois exibe claramente um resultado em que as variáveis do modelo têm efeito sutilmente maior sobre o crescimento econômico municipal do que no estimador MQO, salvo as exceções do parâmetro $\beta$-convergência $\left(\ln y_{0}\right)$ e qualidade institucional $(q)$, cujos efeitos foram menores. Entre as variáveis que tiveram o seu efeito aumentado sobre o crescimento municipal estão taxa de industrialização $(k)$, capital humano $(h)$, investimento em infraestrutura $(i)$, investimento em $\mathrm{P} \& \mathrm{D}$ ( $r$ ) e transferências externas de tecnologias $(t)$.

O regime três possui um coeficiente de convergência ligeiramente superior com um $\beta$ de -0.1601. A maioria dos determinantes de crescimento exibe maior efeito no clube com a maior taxa inicial de educação fundamental. Entre essas variáveis, estão as que representam a formação de capital fixo com a taxa de industrialização $(k)$, investimento público $(g)$, qualidade institucional $(q)$, infraestrutura tecnológica $(i)$, e spillovers tecnológicos $(l)$.

Essa variável threshold é medida pela participação do número pessoas maiores de 25 anos com educação fundamental completa em relação à população municipal no ano de 2000. 
Os clubes de municípios mais avançados são aqueles nos quais o efeito dos investimentos em capital físico e difusão tecnológica são relativamente maiores. Ou seja, embora os municípios do clube de menor taxa de educação tenham maior velocidade de convergência, os municípios de maior crescimento condicional são os que se apropriam em maior medida das vantagens dos investimentos em capital e maiores ritmos de difusão tecnológica. O fato das economias mais desenvolvidas serem mais eficientes na produção com maior produtividade dos fatores e melhor absorção de spillovers tecnológicos é consistente com as previsões do modelo de equilíbrios múltiplos (AZARIADIS; DRAZEN, 1990; DURLAUF, 1993; DOWRICK; GEMMELL, 1991).

A análise threshold estimada fortalece as suposições teóricas de maior efeito dos fatores de produção nas economias mais desenvolvidas. Os determinantes tecnológicos em sua maioria afetam mais fortemente o crescimento daquelas economias com um maior nível de capital humano. Tanto a infraestrutura tecnológica ( $i$ ) quanto a qualidade institucional $(q)$ exercem os seus maiores efeitos nos dois clubes mais desenvolvidos. Nota-se, ainda, que o regime econômico com a menor taxa inicial de educação fundamental exibe um efeito marginal da qualidade institucional zero e da infraestrutura tecnológica inferior ao efeito médio do estimador MQO.

A importação de bens de média e alta tecnologia incorporada é relevante para o desenvolvimento municipal do Brasil, porém afeta exclusivamente os municípios com melhores taxas iniciais de educação fundamental. Esses municípios também são os mais capitalizados e, portanto, demandam mais bens com tecnologia incorporada. Com relação ao diferencial tecnológico, confirma-se a vantagem de absorção dos municípios com maior defasagem tecnológica, porém os maiores efeitos do diferencial tecnológico sobre o crescimento econômico são notados nos regimes de crescimento mais desenvolvidos. No regime um, que corresponde ao grupo mais distante da fronteira tecnológica, nem o diferencial tecnológico nem a transferência tecnológica são significativos. Os spillovers tecnológicos são muito pouco expressivos o que condiz com os resultados encontrados por Falvey, Foster e Greenaway (2007).

\section{Considerações Finais}

A análise de convergência econômica nos municípios brasileiros confirma as vantagens dos estimadores não lineares MMF e threshold sobre o estimador linear segundo os critérios de ajuste e predição. Na análise de convergência com múltiplos equilíbrios, os modelos MMF e threshold mostram que o agrupamento municipal por características comuns a cada grupo aumentam o parâmetro de convergência e o efeito dos determinantes da dotação de fatores e difusão tecnológica, comparativamente ao equilíbrio único. Esse diferencial inerente ao modelo 
de equilíbrios múltiplos sugere que o modelo neoclássico de crescimento regional melhora quando incluídas as diferenças de produtividade na função de produção agregada dos municípios.

O trabalho confirma a existência de múltiplos equilíbrios na evolução do crescimento econômico dos municípios brasileiros, entre 2000 e 2010. A persistência da desigualdade de renda entre municípios se explica pela heterogeneidade regional, diferenças na dotação de fatores de produção, uso eficiente dos bens de capital e difusão tecnológica. A teoria de múltiplos equilíbrios aceita que a formação dos clubes de convergência, avançada e atrasada, é determinada pelas condições iniciais de produção e absorção de tecnologia. Acredita-se que as economias desenvolvidas usem mais eficientemente o capital e as tecnologias disponíveis, perpetuando as desigualdades da renda no tempo.

As duas equações estimadas mostram que, em média, os municípios do Nordeste possuem a pior evolução relativa do crescimento da renda per capita, comparativamente as demais regiões. Esse resultado confirma as evidencias do efeito da heterogeneidade regional no crescimento econômico do país. A ausência de significância das dummies regionais em alguns dos clubes de convergência estimados se deve às baixas disparidades econômicas associadas aos grupos econômicos mais homogêneos.

As desigualdades de renda devidas a diferenças do uso dos fatores de produção e as desigualdades tecnológicas aparecem nos dois estimadores não lineares de equilíbrios múltiplos. A diferença entre elasticidades dos determinantes de crescimento nos clubes de convergência revela que existem diferenças na dotação de fatores e no processo de difusão tecnológica sobre o crescimento econômico dos municípios agrupados. No entanto, modelo MMF não deixa clara a ordenação dos clubes, isso pretere o aspecto dos efeitos das diferenças econômicas no uso mais eficiente dos fatores e/ou maior capacidade de absorção tecnológica em clubes mais desenvolvidos. Já a estimação de clubes de convergência pelo método threshold permite claramente confirmar essas hipóteses, ou seja, o uso eficiente dos fatores de produção e a absorção de spillovers tecnológicos ocorreram principalmente no clube mais desenvolvido (com maior taxa inicial de educação fundamental). O crescimento da indústria, a melhora da qualidade institucional, e o aumento da transferência de tecnologia não afetam significativamente o crescimento econômico do clube mais atrasado (com menor taxa inicial de educação fundamental), mas afetam significativamente o crescimento do clube mais desenvolvido.

Os resultados parecem revelar que os municípios com menores níveis iniciais de educação tendem a entrar em um ciclo de atraso econômico crônico, o que não está univocamente associado a seu menor nível educacional. Outros elementos devem ser considerados. A persistência do atraso pode estar vinculada também a um menor desenvolvimento relativo de suas instituições, à precarieda- 
de das suas infraestruturas e ao baixo nível de formação de sua força de trabalho, o qual dificulta o ritmo da difusão tecnológica. A reduzida capacidade de inovar e absorver conhecimento desses municípios restringe suas possibilidades de crescimento, ampliando, dessa forma, seu atraso econômico em relação aos municípios mais avançados e com maior capacidade de absorção tecnológica.

\section{Referências}

ABRAMOVITZ. Catching up, forging ahead, and falling behind. The Journal of Economic History, v. 46, n. 2, p. 385-406, 1986.

ACEMOGLU, D.; JOHNSON, S.; ROBINSON, J. The colonial origins of comparative development: an empirical investigation. The American Economic Review, v. 91, n. 5, p. 1369-1401, 2001.

AGHION, P.; HOWITT, P. A model of growth through creative destruction. Econometrica, v. 60, n. 2, p.323-351, March 1992.

ANDERSON, G.; LINTON, O.; LEO, T. A polarization-cohesion perspective on cross-country convergence. Journal of Economic Growth, v. 17, n. 1, p. 49-69, 2012.

ANDRADE, E.; LAURINI, M.; MADALOZZO, R.; PEREIRA, P. L. V. Testing convergence across municipalities in Brazil using quantile regression. Insper Working Paper, n. 34, 2002.

ARAÚJO, B. C.; CAVALCANTE, L. R.; ALVES, P. Variáveis proxy para os gastos empresariais em inovação com base no pessoal ocupado técnico-científico disponível na Relação Anual de Informações Sociais (RAIS). Radar IPEA, n. 5, 2009.

AZARIADIS, C.; DRAZEN, A. Threshold externalities in economic development. Quarterly Journal of Economics, v. 105, n. 2, p. 501-526, 1990.

AZZONI, C. Economic growth and regional income inequalities in Brazil. The Annals of Regional Science, v. 35, n. 1, p. 133-152, 2001.

AZZONI, C. R.; MENEZES-FILHO, N.; MENEZES, T. A.; SILVEIRA-NETO, R. Geography and income convergence among Brazilian states. IDB Working Paper, n. 122, 2000.

BAI, J.; PERRON, P. Estimating and testing linear models with multiple structural changes. Econometrica, p. 47-78, 1998.

BARRO, R.; SALA-I-MARTIN, X. Convergence. Journal of Political Economy, v. 100, n. 2, 1992.

BARROS, A. R. Desigualdades regionais no Brasil: natureza, causas, origens e soluções. Rio de Janeiro: Elsevier, 2011. 
BARROS NETO, G.; NAKABASHI, S.L. Relações entre instituições, capital humano e cumulação de capital físico nos municípios brasileiros. Economia ETTecnologia, ano 07, v. 25, abr./ jun. 2011.

BIANCHI, M. Testing for convergence: evidence from non-parametric multimodality test. Journal of Applied Econometrics, n. 12, p. 393-409, 1997.

BREIMAN, L.; FRIEDMAN, J. L.; OLSHEN, R. A.; STONE, C. J. Classification and regression trees. Boca Raton: Chapman and Hall/CRC, 1984.

CAMERON, A. C.; TRIVEDI, P. K. Microeconometrics: methods and applications. Cambridge: Cambridge University Press, 2005.

CASALI, G. F. R.; SILVA, O. M.; CARVALHO, F. M. A. Sistema regional de inovação: estudo das regiões brasileiras. Revista Economia Contemporânea, v. 14, n. 3, p. 515-550, 2010.

CASTELLACCI, F. Closing the technology gap? Review of Development Economics, v. 15, n. 1, p. 180-197, 2011.

CATELA, E. Y. S. Ensaios sobre comércio internacional, tecnologia e crescimento. 2009. Tese (Doutorado) - Programa de Pós-Graduação em Desenvolvimento Econômico, Universidade Federal do Paraná, 2009.

CATELA, E. Y. S.; GONÇALVES, F. Convergência, para onde? Uma análise da dinâmica de distribuição de renda per capita a partir do modelo de misturas finitas. Revista de Economia Aplicada, v. 13, p. 249-275, 2009.

CHEIN, F.; LEMOS, M.; ASSUNCÃO, J. Desenvolvimento desigual: evidências para o Brasil. Revista Brasileira de Economia, v. 61, p. 301-330, 2007.

COE, D. T.; HELPMAN, E.; HOFFMAISTER, A. W. North-South RED spillovers. Economic Journal, v. 107, n. 440, p. 134-150, 1997.

DOWRICK, S.; GEMMELL, N. Industrialisation, catching up and economic growth: a comparative study across the world's economies. The Economic Journal, v. 101, n. 405, 1991.

DURLAUF, S. N. Nonergodic economic growth. Review of Economic Studies, v. 60, n. 2, p. 349-366, 1993.

DURLAUF, S. N.; JOHNSON, P. A. Multiple regimes and cross-country growth behaviour. Journal of Applied Econometrics, v. 10, n. 4, p. 365-384, 1995.

DURLAUF, S.; JOHNSON, P.; TEMPLE, J. Growth econometrics. In: AGHION, P.; DURLAUF, S. (ed.). Handbook of economic growth. Amsterdam: Elsevier, 2004. p. 96.

FALVEY, R.; FOSTER, N.; GREENAWAY, D. Relative ackwardness, absorptive capacity and knowledge spillovers. Economics Letters, v. 97, n. 3, p 230-234, 2007.

FELIPE, J.; FISHER, F. M. Aggregation in production functions: what applied economists should know. Metroeconomica, v. 54, n. 2, p. 208-262, 2003. 
FELIPE, J.; MCCOMBIE, J. S. L. How sound are the foundations of the aggregate production function? Eastern Economic Journal, v. 31, n. 5, p. 467-488, 2005.

FIGUEIREDO, E.; PÔRTO JÚNIOR, S. S. Persistência das desigualdades regionais no Brasil: polarização e divergência. Nova Economia, v. 25, n. 1, p. 195-208, 2015.

FIRME, V.A.C.; FREGUGLIA, R. S. Análise do crescimento dos municípios brasileiros utilizando dados em painel e controles espaciais sobre o modelo de Mankiw, Romer e Weil (1992) para o período de 1980 a 2010. In: ANPEC Nordeste, 18., 2013, Fortaleza. Anais [...] Fortaleza: ANPEC, 2013.

GERSCHENKRON, A. Economic backwardness in historical perspective. Cambridge: Harvard University Press, 1962.

GONÇALVES, E.; RIBEIRO, E. C. B. A.; FREGUGLIA, R. S. Transbordamentos de conhecimento e capacidade de absorção: uma análise para os estados brasileiros. In: ENCONTRO NACIONAL DE ECONOMIA, 38., 2011, Salvador. Anais eletrônicos [...]. Salvador: ANPEC, 2011.

HANSEN, B. E. Threshold effects in non-dynamic panels: estimation, testing, and inference. Journal of Econometrics, v. 93, n. 2, p. 345-368, 1999.

HANSEN, B. E. Sample splitting and threshold estimation. Econometria, v. 68, n. 3, p. 575603, 2000.

HANSEN, B. E. Threshold autoregression in economics. Statistics and its Interface, v. 4, n. 2, p. 123-127, 2011.

HOWITT, P.; MAYER-FOULKES, D. R\&D, implementation, and stagnation: a schumpeterian theory of convergence clubs. Journal of Money, Credit and Banking, v. 37, n. 1, p. 147-177, 2005.

ISIC. Technology intensity definition: classification of manufacturing industries into categories based on RED intensities. OECD: Directorate for Science, Technology and Industry-Economic Analysis and Statistics Division, Revision 3, 2011.

LAURINI, M.; ANDRADE, E.; PEREIRA, P. Clubes de convergência de renda para os municípios brasileiros: uma análise não-paramétrica. In: ENCONTRO BRASILEIRO DE ECONOMETRIA, 25., 2003, Porto Seguro. Anais eletrônicos [...]. Porto Seguro: SBE, 2003.

LUCAMBIO, F. Estimador kernel da função de densidade. Documentos da UFPR - Departamento de Estatística, CE-210, 2008. Disponível em: https://docs.ufpr.br/ lucambio/CE210/ destimator.pdf. Acesso em: 6 ago. 2020.

MANKIW, N.; ROMER, D, W.; DAVID. A contribution to the empirics of economic growth. The Quarterly Journal of Economics, v.107, p. 407-437, 1992.

MARTINHO, M. R. Lei 4.320/64: comentada, em esquemas. [S.n.: s.l.], 2011.

MINISTÉRIO DO PLANEJAMENTO, ORÇAMENTO E GESTÃO (MPOG). Base de dados institucionais dos municípios brasileiros. Brasília, 2000. 
NELSON, R. R.; PHELPS, E. S. Investment in humans, technological diffusion, and economic growth. The American Economic Review, v. 56, n. 2, p. 69-75, 1966.

OWEN, A. L.; VIDERAS, J.; DAVIS, L. Do all countries follow the same growth process? Journal of Economic Growth, v. 14, n. 4, p. 265-286, 2009.

PRESSMAN, S. What is wrong with the aggregate production function? Eastern Economic Journal, v. 31, n. 3, p. 422-425, 2005.

QUAH, D. T. Twin peaks: growth and convergence in models of distribution dynamics. The Economic Journal, v. 106, n. 437, 1996.

RIBEIRO, E. C. B. A. Convergência de renda local entre os municípios brasileiros para o período 2000 a 2005. 2010. Dissertação (Mestrado) - Faculdade de Economia, Universidade Federal de Juiz de Fora, 2010.

ROMER, P. M. Endogenous technological change. Journal of Political Economy, v. 98, part 2, p. 71-102, 1990.

SCHETTINI, D.; AZZON, C. R. Determinantes regionais da produtividade industrial: o papel da infraestrutura. In: DE NEGRI, F.; CAVALCANTE, L. R. (ed.). Produtividade no Brasil: desempenho e determinantes. Brasília: IPEA, 2015. v. 2.

SCHETTINI, D.; AZZON, C. R. Productive efficiency and the future of regional disparities in Brazil. Nova Economia, v. 28, n. 2, p. 347-384, 2018.

SCHIOPU, I. Technology adoption, human capital formation and income differences. Journal of Macroeconomics, v. 45, p. 318-335, 2015.

SOLOW, R. M. A contribution to the theory of economic growth. The Quarterly Journal of Economics, v. 70, n. 1, p. 65-94, Feb. 1956.

SOLOW, R. M. Technical change and the aggregate production function. The Review of Economics and Statistics, v. 39, n. 3, p 312-320, Aug. 1957.

URRACA-RUIZ, A.; LAGUNA, N. Convergence and Stages of Technical Change. In: INTERNATIONAL GLOBELICS CONFERENCE, 12., 2014, Etiopia. 


\section{Apêndice A - Modelo Teórico Neoclássico Integrado}

Considerando-se a existência de um ambiente com inovação e difusão tecnológica, os múltiplos equilíbrios podem representar situações transitórias cuja dinâmica implica potenciais catching up. A transferência tecnológica desde as economias líderes para as seguidoras, através do processo de difusão, estimula, principalmente, a produtividade das economias mais atrasadas. Entretanto, a transferência tecnológica pressupõe a capacitação prévia das economias seguidoras para absorver e empregar eficientemente as tecnologias disponíveis. A escalar tecnológica da função de produção neoclássica foi modelada para compreender a absorção de spillovers tecnológicos $\left(S_{j t}\right)$ :

$$
A_{j t}=\pi A_{j t-1}^{\xi} S_{j t}
$$

O valor de $\left(S_{j t}\right)$ vem determinado pelas condições para a difusão apresentadas pela economia, o que inclui infraestrutura tecnológica $\left(I_{j t}\right), \mathrm{P} \& \mathrm{~B}\left(R_{j t}\right) \mathrm{e}$ qualidade das instituições $\left(\mathrm{Q}_{\mathrm{j}}\right)$ (ROMER, 1990; AGHION; HOWITT, 1992; ABRAMOVITZ, 1986; GERSCHENKRON, 1962), além dos fatores externos resultados de acordos de cooperação internacional $\left(\mathrm{F}(\mathrm{X}){ }_{\mathrm{j} t}\right.$ ) (GONÇALVES; RIBEIRO; FREGUGLIA., 2011; FALVEY; FOSTER; GREENAWAY, 2007).

$$
S_{i t}=\left(\frac{y_{f}-y_{j}}{y_{f}}\right)^{\tau_{1}} \varphi_{j t}, \text { para } \varphi_{j t}=I T_{j t}{ }^{\varphi_{1}} R_{j t}^{\varphi_{2}} Q_{j t}^{\varphi_{3}} F(X)_{j t}
$$

A função vem determinada pela transferência externa de tecnologias de alto conteúdo tecnológico através da importação de máquinas e equipamentos intensivas em $\mathrm{P} \& \mathrm{D}$ e pelos spillovers externos de conhecimento representando o conhecimento codificado contido nas máquinas e equipamentos importados. Portanto:

$$
F(X)_{j t}=\left(\frac{y_{f}-y_{j}}{y_{f}}\right)^{\tau_{2}} w_{j t}, \text { para } w_{i t}=T_{j t}{ }^{w_{1}} L_{j t}^{w_{2}}
$$

Substituindo-se as equações 9 e 10 na escalar tecnológica do modelo Solow ampliado, tem-se:

$$
A_{i t}=\pi A_{j t-1}^{\xi}\left(\text { gap }_{j t}\right)^{\tau_{1+} \tau_{2}} I_{j t}{ }^{\varphi_{1}} R_{j t}^{\varphi_{2}} Q_{j t}^{\varphi_{3}} T_{j t}{ }^{w_{1}} L_{j t}^{w_{2}}
$$

na qual a soma dos expoentes $\left(\tau_{1}+\tau_{2}=\tau\right)$ é o coeficiente de spillover tecnológico da defasagem tecnológica $\left(g a p_{j t}\right)$ dado pela diferença relativa de produtividade entre a fronteira tecnológica $\left(y_{f}\right)$ e as economias seguidoras $\left(y_{j}\right)$. Assumindo-se, então, que o gap $_{j t}=1-\left(y_{j} / y_{f}\right)$ e que $\pi$ é uma constante positiva, o logaritmo da função de progresso tecnológico é:

$$
\ln A_{j t}=\ln \pi+\xi \ln A_{j t-1}+\tau \ln \left(g a p_{j t}\right)+\varphi_{1} \ln I_{j t}+\varphi_{2} \ln R_{j t}+\varphi_{3} \ln Q_{j t}+\cdots . .+w_{1} \ln T_{j t}+w_{2} \ln L_{j t}+\ln u_{j}(12)
$$


Substituindo-se o logaritmo do progresso tecnológico na função de produção per capita em logaritmo, chega-se à equação completa com progresso tecnológico endógeno:

$$
\begin{gathered}
l n y_{j t}=\alpha \ln k_{j t}+\theta \operatorname{lng} g_{j t}+(1-\alpha-\theta-\delta)\left(\ln \pi+\xi \ln A_{j t-1}+\tau \ln \left(\operatorname{gap}_{j t}\right)+\varphi_{1} \ln I_{j t}+\right. \\
\left.\varphi_{2} \ln R_{j t}+\varphi_{3} \ln Q+\varphi_{4} \ln I_{j t}+w_{1} \ln T_{j t}+w_{2} \ln L_{j t}\right)+\left(\ln u_{i t}+\ln e_{j t}\right)
\end{gathered}
$$

Derivando-se o logaritmo da função de produção em relação ao tempo com spillovers tecnológicos explícitos, chega-se à equação do crescimento econômico endógeno com capital humano, investimento em pesquisa $\left(\hat{r}_{j}\right)$ e absorção de spillovers tecnológicos. O termo de erro é a soma do logaritmo natural do distúrbio aleatório das funções de produção a escalar tecnológica:

$\hat{y}_{j}=\alpha \hat{k}_{j}+\theta \hat{g}_{j}+\delta \hat{h}_{j}+(1-\alpha-\theta-\delta)\left(\xi \hat{A}_{j t-1}+\tau g \widehat{a}_{j}+\varphi_{1} \hat{i}_{j}+\varphi_{2} \hat{r}_{j}+\varphi_{3} \widehat{a}_{j}+w_{1} \hat{t}_{j}+w_{2} \hat{l}_{j}\right)+\mu_{j}(14$

O valor $(1-\alpha-\theta-\delta)\left(\xi \hat{A}_{j t-1}\right)$ corresponde à constante $(c)$ da equação 16 , que representa o crescimento que ocorre, mesmo não havendo formação de capital fixo, humano e qualidade institucional ou absorção de spillovers. Os demais parâmetros da equação são determinados pelas respectivas multiplicações:

$$
\begin{aligned}
& (1-\alpha-\theta-\delta) \tau=\emptyset_{1} ;(1-\alpha-\theta-\delta) \varphi_{1}=\emptyset_{2} ;(1-\alpha-\theta-\delta) \varphi_{2}=\emptyset_{3} ; \\
& (1-\alpha-\theta-\delta) \varphi_{3}=\emptyset_{4} ;(1-\alpha-\theta-\delta) w_{1}=\emptyset_{5} ;(1-\alpha-\theta-\delta) w_{2}=\emptyset_{6}
\end{aligned}
$$

Então, a equação 13 passa a ser notada assim:

$$
\hat{y}_{j}=c+\alpha \hat{k}_{j}+\theta \hat{g}_{j}+\delta \hat{h}_{j}+\emptyset_{1} \widehat{g a p_{j}}+\emptyset_{2} \hat{i}_{j}+\emptyset_{3} \hat{r}_{j}+\emptyset_{4} \hat{q}_{j}+\emptyset_{5} \hat{t}_{j}+\emptyset_{6} \hat{l}_{j}+\mu_{j}
$$

Como as elasticidades de produção dos fatores de formação de capital fixo tomam valores entre zero e um, é possível que $1-\alpha-\theta-\delta \leq 0$. Se o resultado dessa subtração for zero, o efeito do progresso tecnológico sobre o crescimento econômico se torna nulo. Por outro lado, quanto menores forem as elasticidades $\alpha, \theta$ e $\delta$, maiores serão as elasticidades $\left(\emptyset_{1}, \emptyset_{2}, \emptyset_{3}, \emptyset_{4}, \emptyset_{5} e \emptyset_{6}\right)$ dos determinantes $g \widehat{a p}_{j}, \hat{k}_{j}, \hat{r}_{j}, \hat{q}_{j}, \hat{t}_{j} e \hat{l}_{j}$ sobre o crescimento econômico. Esta última equação pode ser agrupada em dois vetores, $x$ e $w$, em que $x$ representa o vetor de dotação fatorial (capital físico e humano) e $w$, o vetor dos determinantes tecnológicos (capacidade de absorção, spillovers e difusão tecnológica).

$$
y=\beta x+\delta w+\mu_{j}
$$

Essa especificação permite isolar a diferente natureza de cada um dos determinantes e diferenciar seu papel no processo de convergência em clubes (CASTELLACI, 2011; URRACA-RUIZ; LAGUNA, 2014). A partir da hipótese de que os diferenciais tecnológicos explicam as desigualdades econômicas - e que é necessário um certo nível de capacitação para fazer possível a absorção tecnológica -, 
a difusão das tecnologias externa de alto conteúdo tecnológico poderia ter dois efeitos contrapostos sobre o crescimento municipal: por um lado, a transferência de tecnologia das regiões líderes implicaria um aumento de produtividade dos fatores, reduzindo o diferencial de produtividade; porém, por outro lado, a melhor capacidade inicial de absorver e empregar eficientemente as tecnologias externas disponíveis poderia levar a uma ampliação das desigualdades regionais (NELSON; PHELPS, 1966; SCHIOPU, 2015). Esse efeito teria como consequência o que se conhece por erosão da capacidade de absorção das tecnologias (HOWIT; MAYER-FOULKES, 2005).

\section{Apêndice B - Índice de Qualidade Institucional dos Municípios}

O índice de qualidade institucional $\left(q_{j}\right)$ no nível municipal (j) foi construído a partir da média aritmética de três subíndices que recolhem três dimensões da qualidade das instituições $\left(s_{i j}\right)$ : participação política da sociedade, capacidade financeira, e capacidade gerencial do município:

$$
q_{j}=\frac{1}{3} \sum_{i=1}^{3} s_{i j}
$$

Cada $\mathrm{S}_{\mathrm{ij}}$ é composto por uma média ponderada $\left(\mathrm{p}_{\mathrm{r}}\right)$ de um conjunto de microíndices $(m)$ que definem os aspectos institucionais $(r)$. Cada $m$ recebe o peso que se julga representativo para cada vertente institucional dos $\mathrm{S}_{\mathrm{ij},}$ Os pesos variam entre $4 \%$ e $11 \%$ em função dos julgamentos de maior e menor importância na composição do índice (ver Figura 1). 
Figura 1 - Indicador de qualidade institucional dos municípios brasileiros em \%

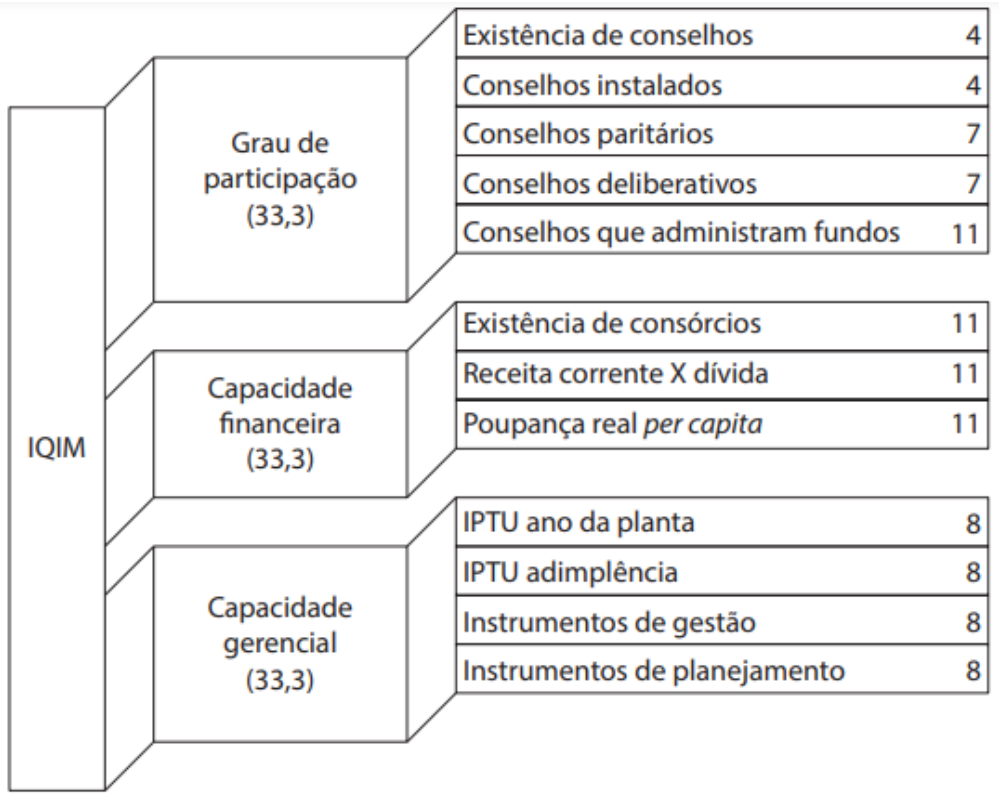

Fonte: Agenda Política Institucional (MPOG, 2000).

\section{Autor correspondente:}

Sergiany da Silva Lima

E-mail: sergiany.lima@ufrpe.br
Recebido em: 16/04/2018.

Aceito em: 03/06/2019.

\section{(cc) BY}

